\section{Sean Kelly}

was one of the very first designers of an enterprise data warehouse and is the author of Data Warehousing

- The Route to Mass

Customisation (1993; John

Wiley \& Sons). He has continued to bridge the gap between data warehouse technology and the business applications of information and has developed both a data warehouse project methodology and a customer relationship management framework for data warehouse data architectures. He founded the Data Warehouse Network in 1994 and this company has evolved to become Europe's premier data warehouse design consultancy group. Data Warehousing in Action (Wiley) was published in 1997 and Sean is currently finalising The Information Wars, a book for business leaders which draws on his considerable experience of bringing organisations into the age of information exploitation.
Keywords: Loyalty, profitability, cross-selling, up-selling, customer relationships, data warehouse

Sean Kelly

Sybase Business Intelligence,

Crown Lane,

Maidenhead,

SL6 8QZ

Tel: +44 1628597100

e-mail:kelly@sybase.com

\section{Analytical CRM: The fusion of data and intelligence}

\author{
Sean Kelly \\ Received (in revised form) 19th October, 1999
}

\begin{abstract}
A good deal has been written about customer relationship management (CRM), one-to-one marketing and event-driven marketing, and the dramatic potential of these strategies is reasonably clear to all. However, the deployment of the necessary information systems to realise the marketing nirvana of genuine customer-centred enterprises is still a good way off. There is one single overwhelming reason why progress is slow: data is not being harnessed with anything like the degree of sophistication that technology allows. It may even be, from a casual observation of the enterprises that trumpet a CRM strategy, that many fundamentally misunderstand the concept. For a customer-centred strategy to be successful we need to comprehensively understand customer behaviour and responsiveness. This, in turn, will allow us to understand customer life cycle, customer loyalty, customer risk, customer profitability and customer segmentation. From here we may proceed to a corporate strategy based on this intelligence; a strategy that will undoubtedly precipitate a radical overhaul of business processes and culture. The systems associated with this customer discovery process are termed 'analytical CRM', in contrast to the operational CRM systems that are deployed in a call centre to handle the interaction with the customer. Many enterprises have invested heavily in the infrastructure for operational CRM (call centre technology, funnel management systems, campaign management technology, loyalty cards) without having any coherent strategy for analytical CRM. The result is that they are well positioned to deal with individual customer interactions but hopelessly blind to the history and pattern of customer transactions over time. The outcome is, generally, disappointing and predictable. The source of failure can be located in the contradiction of attempting to deal with customers as distinct individuals without retaining a corporate memory of those individuals.
\end{abstract}

\section{The CRM elixir}

Many commentators and consultants in the field of marketing are busily selling business executives with the necessity for CRM. Very often, what is not adequately explained are the steps that are required to transition a mass-marketing culture to a business environment for one-to-one marketing. It is even rarer to hear, on the business speaking circuit, any insight into the complexity of the information architecture that must first be created in order to realise the vision of mass customised selling. The 


\section{One-to-one marketing is a radically revised business process}

\section{The customer is a far more stable anchor for the business than are products}

\section{Each customer is unique}

\section{Customers want to buy time}

\section{Customer behaviour is totally predictable}

net result is an expectation on the part of many business executives that one-to-one marketing is simply a fashionable business strategy rather than a radically revised business process.

The test of a business that has genuinely embraced CRM is whether the focus of the business has moved from product to customer. In the traditional business model the critical issue was if the product (which was perceived as tangible and stable) could be sold to customers (who were perceived to be spectral and fickle). The mass customisation revolution turns this assumption on its head. The CRM enterprise makes its most significant investment in the customer, and products become simply things that need to be devised and sourced in response to customer needs. When an enterprise invests time, money and resources into understanding customer behaviour it quickly becomes apparent that the customer is a far more stable anchor for the business than are products. The days when we could regard customers as a renewable resource that could always be depended on to exhibit a Pavlovian response to the stimuli of price and convenience have all but disappeared.

So, what does our experience of analysing information about customers tell us?

- First, each customer is unique and there is no such thing as a typical customer or even typical profiles that might be convertible into segments (which rules out focus groups, statistical sampling or geodemographic profiling as adequate techniques to understand or anticipate customer behaviour).

- Secondly, customers expect the supplier enterprise to retain a memory of their transactions and are enraged by organisations that repeatedly ask them for the same information. (How many of us get asked every time we check into the same hotel chain if we want a non-smoking room? ... We grit our teeth and tell them for the umpteenth time, knowing they will discard this information as soon as we check out.)

- Thirdly, the most attractive product that consumers want to buy is time. (Why trudge around a supermarket putting the same branded products in your trolley each week when your grocer could do it for you?)

- And finally, customer behaviour is totally predictable. Every customer action (whether it represents a threat or opportunity for the enterprise) is preceded by telltale warning signs hidden in the mass of data.

What is also apparent is that customers clearly understand the potential of technology much better than do many business leaders. When you hand a customer a loyalty card you immediately create the expectation in the mind of the customer that you are now going to study their behaviour. That is the implicit contract that is created with the customer. In the process of accepting the card they permit their behaviour to be scrutinised. In return they expect the supplier to do something intelligent with the information. The same principle applies to call centres and electronic commerce. Once the customer realises that he or she is not an 
Kelly

\section{Playing with CRM is dangerous}

\section{Integrated perspective on sales}

\section{Facilitates the building of genuine customer relationships}

anonymous shopper, they lose all patience with being treated like one. Which is why playing with CRM is dangerous. Trapping individual customer events in an environment where the data are subsequently discarded, hopelessly fragmented or intact but unanalysed is a recipe for customer disillusionment on a grand scale.

\section{Key analytical CRM applications}

\section{Sales analysis}

The sales analysis application provides an analysis of sales by different dimensions, including sales by channel/outlet or organisational unit; sales by product, product category or product group; sales by region; sales by season; cross-selling; up-selling; sales by customer, customer segment or customer demographics; an analysis of the relationship between customer satisfaction and sales volumes; an analysis of the characteristics of customers who purchase particular products; and an analysis of the mix of products sold. This application offers the organisation an integrated perspective on sales and enables the sales function to understand the underlying trends and patterns in the sales data.

\section{Customer profile analysis}

This application allows the organisation to distinguish in the mass of customers the individual customer as well as the many micro-segments that make up the whole. Increasingly customer segmentation is forming an essential element of marketing strategy as markets become more fragmented and customer segments exhibit distinct and different characteristics. This application facilitates the building of genuine customer relationships in the era of one-to-one marketing. Customer profile analysis includes an analysis of both households and individual customers. The analysis includes profiling by product utilisation and frequency; by product holding pattern; by demographic characteristics; by psychographic characteristics; and by geographic characteristics.

\section{Campaign analysis}

Being able to target customers accurately in campaigns and promotions and being able to analyse the response to a promotion episode are key parts of the learning process that enables the transition from mass marketing to mass customisation. Most organisations launch many different kinds of promotional campaign for many different products in many different media. This application enhances the organisation's understanding of the entire process, from selecting those customers to be targeted to analysing how they responded. It is clearly an analytical application and is a necessary prerequisite for any operational software packages designed to manage the campaign episode itself. The campaign analysis application includes an analysis of both households and individual customers, and an analysis of customers who are highly responsive to campaigns; it compares the characteristics of those customers who are highly unresponsive and the characteristics of customers who have opted to be excluded from campaigns; and it provides the ability to measure the 
Loyalty analysis

measures and monitors customer loyalty and facilitates customer retention

\section{Analysing customer contracts is an essential ingredient in maintaining and nurturing customer relationships} (1) (1)

effectiveness of individual campaigns and the effectiveness of different media, and to conduct cost-benefit analyses of campaigns.

\section{Loyalty analysis}

One of the keys to profitability in any enterprise is customer loyalty. Yet few organisations measure customer loyalty in a structured way or seek to understand the underlying causes of customer attrition. This application is designed to do just that. The loyalty analysis application measures customer loyalty with reference to the duration of the customer relationship; the range of services and products consumed by the customer; the frequency of purchases by the customer; the characteristics of loyal and disloyal customers; the rate of customer attrition; the extent of customer attrition; seasonal and cyclical factors that impact on customer attrition; the demographic, psychographic and geographic influences on customer attrition; and the attrition rates of customer in respect of individual products. By itself the loyalty analysis application measures and monitors customer loyalty and facilitates the development of customer retention programmes. When combined with the full suite of applications the loyalty of customers can be assessed in the context of their value, their contact history, the segments they belong to and individual transaction events that may influence their loyalty.

\section{Customer contact analysis}

Customers interact with organisations in many ways using different touchpoints to initiate enquiries, make complaints, tender compliments, provide feedback, report faults or make suggestions. This information provides valuable insights into the behaviour of the customer and the track record of the organisation servicing the customer. Identifying the likely level of satisfaction or dissatisfaction of a customer can be clearly seen in the customer contact history of that individual. This application provides the capability to analyse the customer contact history of individual customers and study resolved and unresolved complaints; the number of positive and negative contacts; the time taken to resolve customer complaints and enquiries; and the response of customers to these interactions. Analysing customer contacts is an essential ingredient in maintaining and nurturing customer relationships and preserving the loyalty of customers into the future.

\section{Profitability analysis}

In any organisation it is essential to understand profitability in order to be able to determine pricing, award discounts, allocate resources or develop strategy. But profitability is a many-faceted concept and can be considered in the context of an organisation, an asset, a channel, a product, a product

\author{
Essential to \\ understand \\ profitability in all its \\ dimensions
} category, a brand, a customer or a customer segment. In addition to these dimensions of profit, most organisations will wish to measure gross profits, net profits and margins. To measure and analyse the many different dimensions of profitability is the objective of this application. 
Kelly

Analytical CRM the missing link

\section{Conclusions}

What the present state of the CRM marketplace demonstrates is that there is a missing link, and that link is analytical CRM. Operational CRM systems are well developed and are now deployed in many organisations to handle campaigns and customer interactions. But very often this capability allows the enterprise to achieve no more than more sophisticated mass marketing. And real breakthroughs cannot be achieved in establishing customer relationships until the mass-marketing paradigm is broken. Simply being able to observe customer responses to campaigns merely measures the success of that campaign. It does not tell you if it was the right campaign. And clearly it provides no basis for one-to-one marketing. The source of intelligence for segmentation, targeting and the pursuit of one-to-one marketing lies in the fragmented mass of customer interactions that are captured on the many different operational systems. Insights into customer behaviour cannot be derived solely from the interactions they have with the company call centre, but are betrayed by the behaviour that can be discerned from the billing systems, the account management systems, the electronic point-of-sale systems, the financial systems and the administrative systems of the enterprise. Until these data are harnessed, the marketing department is still flying blind. Undeniably, harnessing these data is a greater challenge than simply deploying a system to manage the campaign and sales process. Until a business can predict future loyalty and profitability it has no basis for really managing the business. Equating sales force automation or campaign management with CRM is naïve and will, ultimately, alienate customers who, increasingly, have a very clear understanding of what constitutes a relationship. 


\section{The many CRM analytical applications required by the enterprise should be combined in an integrated architecture}

\section{The particular and different source data in each vertical industry must be reflected in industry- specific data warehouse design for analytical CRM}

\section{To analyse the behaviour of individual customers requires detailed transacton histories of each individual customer}

\section{Ease of navigation and query performance can only be satisfied by using dimensional design structures}

\section{The first rule of analytical CRM:}

Application integration

Let us consider the example of sales data. These 'fact' data describing the sale transactions are the source of many different kinds of analytical application required by the enterprise. They provide the means of tracing responsiveness to promotions and campaigns. They are the raw material to explore opportunities for cross-selling and up-selling. They are the basis for monitoring customer loyalty and attrition. They provide the key to unlocking the secrets of profitability. Sales data are an important ingredient in the analysis of risk. And the seasonal, cyclical, geographic, and affinity patterns of sales unmask for us the segments in our marketplace. So, what conclusions can we draw from this? Foremost is the conclusion that the architecture required for these different applications is a common architecture, and to develop these applications separately and independently would be architectural madness.

The second rule of analytical CRM: Industry specificity

It is abundantly clear that the market for analytical CRM cannot be served by generalised horizontal solutions or by 'vanilla' applications that are applicable to all industries. The 'fact' data that describe customer loyalty in an insurance company (eg quotations, renewals, claims, cancellations) are fundamentally different from the transaction facts that describe a customer of a telephone company (telephone traffic, faults, provisioning). Any attempt to devise a vanilla solution that could be applied to both could only survive at a hopelessly high level of abstraction. In this regard the market for packaged solutions for CRM will be different from the ERP market (enterprise resource planning — integrated software solutions that address human resource, finance and logistics functions) where the business processes being supported (finance, human resources, logistics) are broadly similar from one corporation to another.

The third rule of analytical CRM:

Data atomicity

One of the lessons of one-to-one marketing is that the real insights into customer behaviour are to be found in the detailed transaction-level data that describe that customer's behaviour. Data that are summarised and consolidated are often misleading and always inflexible. The trends and patterns of customer behaviour that can be unmasked are hidden in the mass of transaction detail.

The fourth rule of analytical CRM:

Dimensionality

When attempting to design an information architecture that models the data for analytical CRM applications it is clear that the traditional 3NF (third normal form, a design technique used for online transaction systems) design techniques are inappropriate; dimensional design structures are now used by all experienced data warehouse designers. The organisation of data into 'facts' and 'dimensions' boosts query performance, improves navigation and eases the task of maintaining the warehouse. 